\title{
EFFECT OF FORCED CONVECTION HEAT TRANSFER OVER SIDE-BY-SIDE SQUARE CYLINDERS IN A STEADY CONFINED FLOW REGIME
}

\author{
ANIRUDDHA SANYAL \\ Department of Chemical Engineering \\ Indian Institute of Technology Roorkee \\ Roorkee 247667, Uttarakhand, India \\ saniruddhaan@gmail.com \\ Amit Dhiman \\ Department of Chemical Engineering \\ Indian Institute of Technology Roorkee \\ Roorkee 247667, Uttarakhand, India \\ dhimuamit@rediffmail.com, amitdfch@iitr.ac.in
}

[Received: April 16, 2016, Accepted: August 12, 2016]

\begin{abstract}
A two-dimensional numerical study has been conducted to analyze forced convective confined flow and heat transfer from a pair of side-by-side square cylinders with a transverse gap ratio (defined as the ratio of the distance between the obstacles to the size of an obstacle) of 1.5. The flow is steady Newtonian in a viscous dominant flow field, investigated at Reynolds numbers $(R e)$ ranging from 10 to 40 for Prandtl numbers $(P r)=0.7$ and 50. The present results are found to be in the excellent agreement with the literature, with a maximum deviation of $1.5 \%$. It is observed from the streamlines that the wake formation increases with $R e$. The onset of flow separation was observed at $R e=7$ and further a correlation connecting wake length with $R e$ is stated.
\end{abstract}

Mathematical Subject Classification: 05C38, 15A15

Keywords: Drag coefficient, Nusselt number, side-by-side configuration, square bluff bodies, wake length

\section{Nomenclature}

$\beta \quad[-] \quad$ ratio of the side of one square cylinder to the channel height (called here blockage ratio),

$\rho \quad\left[\mathrm{kg} / \mathrm{m}^{3}\right] \quad$ fluid density,

$\mu \quad[\mathrm{Pa} s] \quad$ viscosity of the fluid,

$\tau \quad[\mathrm{Pa}] \quad$ shear stress,

$C_{D} \quad[-] \quad$ drag coefficient,

$C_{p}$ [- ] pressure coefficient,

$F_{D} \quad[\mathrm{~N} / \mathrm{m}] \quad$ drag force per unit length of the obstacle,

$H \quad[\mathrm{~m}] \quad$ transverse height of the domain, 


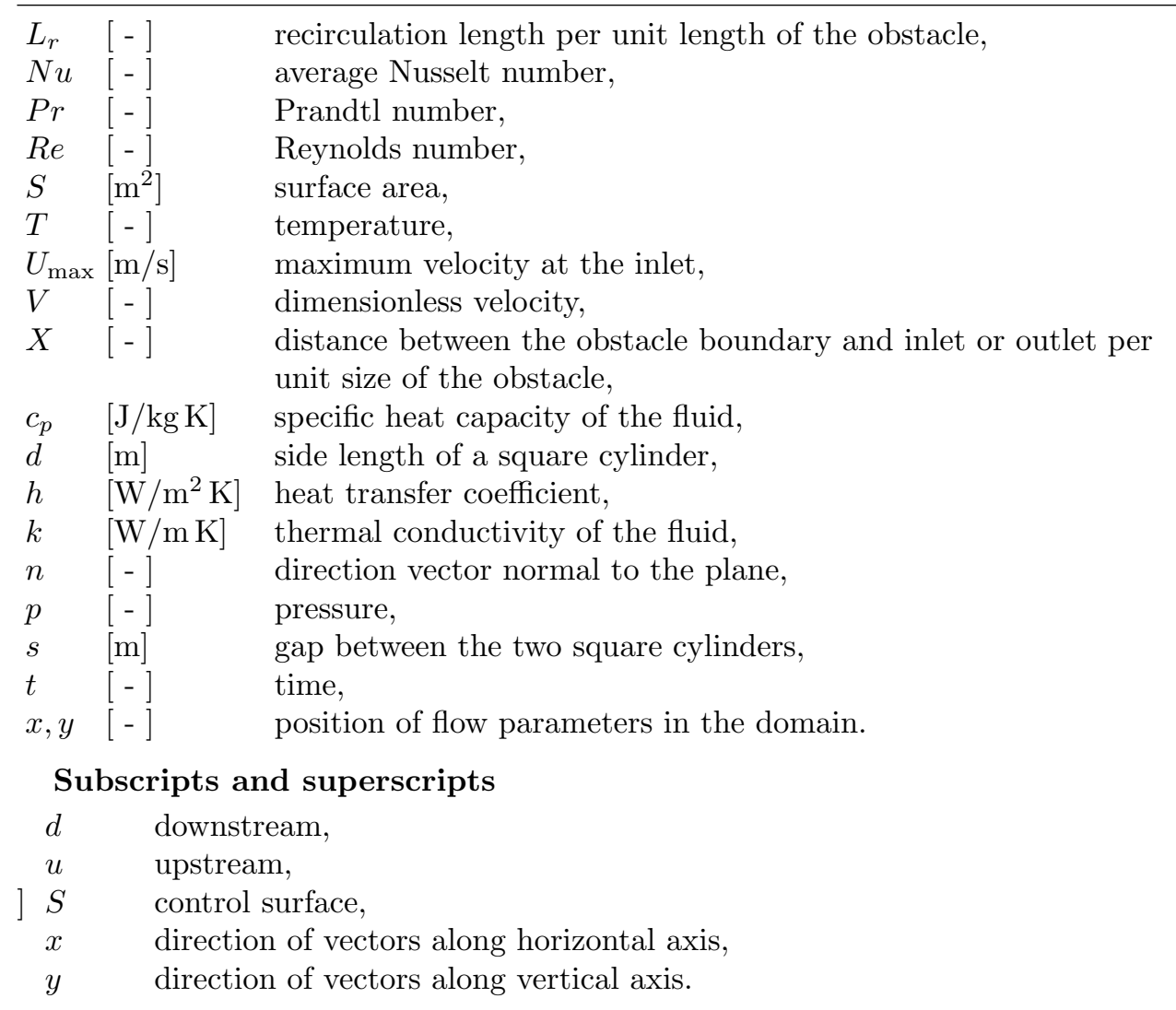

\section{INTRODUCTION}

Ever since the inception of study for fluid flow and heat transfer past a bluff body in a confined domain, decades have passed for research mainly on an experimental basis, which has incurred huge cost. As a result several attempts have been made to analyze this process through numerical modeling and simulation. Modern numerical methodologies like finite volume method (FVM), lattice Boltzmann method (LBM), optical density method, etc. have profound applications in decoding the sets of complicated partial differential equations which define the flow and heat transfer processes. This field has heavy application in process heat transfer equipment, structural dynamics and mechanical, chemical and other related engineering applications.

When two or more bluff bodies are placed in proximity, the intricacy in predicting momentum and heat transfer around it is aggravated and interference effects are severe. As a result, the flow and thermal patterns differ from those of a single circular cylinder.

Valencia and Paredes [1] performed a numerical study to examine the flow and heat transport characteristics in a plane channel with two square cylinders (or square bars) placed side-by-side to the impending flow for a transverse gap ratio $(s / d)$ ranging from 
0 to 5 for Reynolds number Re varying from 25 to 125 at a constant blockage ratio $\beta$ of $12.5 \%$. The mathematical outcomes divulge the complicated formation of the flow. The flow remains steady at $R e=200$; whereas, periodicity or unsteadiness is observed as the Re is further increased. Peng [2] studied the fluid flow past two sideby-side square bluff bodies with a constant gap ratio of 2 in an unconfined domain by both mathematical simulation as well as investigational flow-visualization methods at $R e=100$. Bi-stable flows were observed, with both in-phase and anti-phase synchronized patterns, as a result of adjusting the initial conditions. Agrawal et al. [3] examined the flow over two side-by-side square cylinders using the LBM, identified regimes with both synchronized and scattered vortex formation, and determined the type of vortex shed from the square cylinder in either regime for a uniform flow field at the upstream. Numerical outcomes for two gap ratios of 0.7 and 2.5 for the fixed $R e=73$ and blockage of $5.55 \%$ had been reported. Later, Rao et al. 4 carried out an extension work of Agrawal et al. 3] and performed a quantitative study of the flow over two side-by-side square bluff bodies, for transverse gap ratio varying from 1 to 2.7 and $R e$ varying from 73 to 200 at the blockage of $5.55 \%$. They revealed that for a transverse gap ratio lesser than 1.5, the flow demonstrates a flip-flop behavior known as chaotic; however, for $s / d$ greater than 1.5 , the flow tends to synchronize, known as quasi-periodic and for $s / d>4$ synchronized flow was observed. The transition between chaotic and quasi-periodic regimes occurs at $s / d=1.5$. Durga Prasad and Dhiman [5] analyzed the steady and unsteady laminar flow and heat transfer in a confined domain for a pair of side-by-side square cylinders for $R e=10$ to 100 at $\operatorname{Pr}=0.7$ to 50 with the gap ratio from 1.5 to 10 in a transverse domain height of $18 d$ [3, 4]. It was shown that the overall drag coefficient decreases with increasing $R e$ and $\operatorname{Pr}$ for all values of gap ratios. The enhancement in average Nusselt number was found more than $76 \%$ for the range of settings covered. They also found the occurrences of in-phase and anti-phase flow past the square cylinders at various $R e$. They also found that beyond a gap ratio of 2.5 the steadiness in flow was observed till $R e<60$ which was limited to $R e=50$ for a smaller gap ratio.

Furthermore, sufficient information is now available in the literature on the flow around two side-by-side square cylinders in the turbulent regime. For instance, Wong et al. [6], Kolar et al. [7] and Alam et al. 8] executed experimental investigation of the wake formation around a pair of side-by-side square cylinders. Harichandan and Roy [9] displayed the strong dependence of flow characteristics on the transverse gap ratio and $R e$, with the former being more dominant than the latter.

Mizushima and Akinaga 10 studied wake interactions in the flow past a row of square bars by both numerical replication and experimental determination on the postulation that the flow is two-dimensional $(2 D)$, incompressible. Kumar et al. [11] reported the presence of synchronous, quasi-periodic, and chaotic flow regimes for $s / d$ ranging from 0.3 to 12 for nine square cylinders in side-by-side arrangement at $R e=80$. Along the same line, Sewatkar et al. [12] determined the effects of transverse gap ratio and $R e$ on the flow around a row of cylinders for $R e$ ranging from 30 to 140 and $s / d 1$ to 4 . Chatterjee et al. 13] executed numerical simulation for the flow 
around a row of five square bluff bodies kept at a side-by-side display for transverse gap ratios of $1.2,2,3$, and 4 at $R e=150$.

Thus, from the foregoing argument, one can summarize that no work is available on the onset of flow separation and the wake length at varying $s / d$ in the confined flow around two side-by-side square cylinders in a cross flowing domain. Because the multiple bluff obstacles create a complex flow and thermal structures even at low Reynolds numbers, and owing to the engineering relevance in various applications (compact heat exchangers, plate type heat exchangers, etc.); the analysis of flow and thermal prototype is necessary. A close look at momentum and heat transfer processes in a highly viscous force dominated steady laminar flow regime $(10=R e=40)$ inside a confined domain has been attempted. It is also seen that very few papers have mentioned the occurrence of a recirculation zone quantitatively. Hence, an attempt has been made to study recirculation length from the flow field and domain parameters $R e=10$ to $40, \operatorname{Pr}=0.7,50$ and $s / d=1.5$.

\section{Mathematical formulation and solution methodology}

Any flow problem involves sets of partial differential equations which need to be solved using some numerical techniques. The sequence of approaching the solution methodology involves initial statement of the problem followed by mention of the governing equations coupled with its boundary conditions. Further, generation of an optimal grid is solved here using ANSYS FLUENT. The basics regarding these topics are well explained in Chabra and Richardson [14].

3.1. Problem statement. The following problem has been assumed to be a simplified case of the flow of a fluid past a pair of square cylinders in a $2 D$ domain.

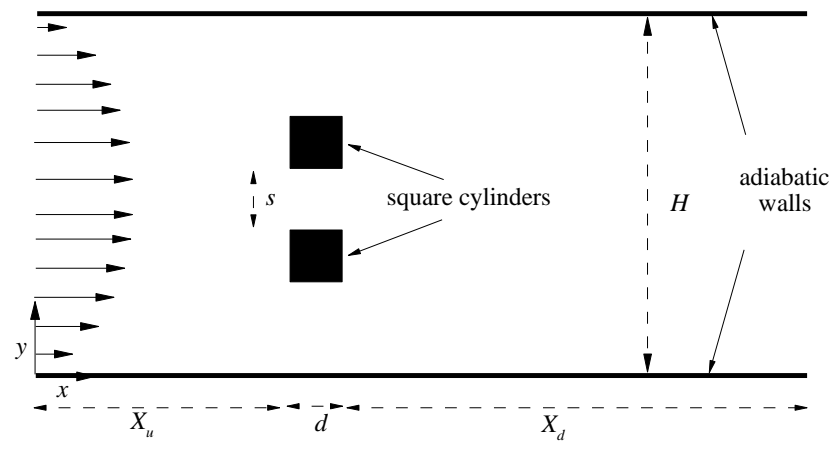

Figure 1. Schematic diagram for fluid flowing past side-by-side square cylinders 
Here, the square cylinders are assumed to be infinitely long and perpendicular to the main flow. The variation of physical process parameters along this direction is zero till Re about 150. Following Durga Prasad and Dhiman [5, the domain has been set in a standardized format, where the upstream distance from the square cylinders, $X_{u}$ is set at 8.5 and downstream distance, $X_{d}$ as 16.5 . The side length of the square cylinder, $d$ is set as unity. The transverse gap ratio, $s / d$, is taken as 1.5.

Figure 1 aptly depicts the aforesaid problem statement. The $2 D$ approximation is well established in a highly viscous force dominant flow field along with a small thermal gradient which is significant enough to catch the change in flow and thermal patterns due to flow around a pair of side-by-side square cylinders with good numerical accuracy.

3.2. Governing equations. For a $2 D$ convective laminar flow, the corresponding dimensionless equations are:

Continuity equation for incompressible fluid flow

$$
\frac{\partial V_{x}}{\partial x}+\frac{\partial V_{y}}{\partial y}=0
$$

Momentum equations neglecting the body forces

$$
\begin{aligned}
& \frac{\partial V_{x}}{\partial t}+\frac{\partial V_{x} V_{x}}{\partial x}+\frac{\partial V_{y} V_{x}}{\partial y}=-\frac{\partial p}{\partial x}+\frac{\partial 1}{R e}\left(\frac{\partial^{2} V_{x}}{\partial x^{2}}+\frac{\partial^{2} V_{x}}{\partial y^{2}}\right), \\
& \frac{\partial V_{y}}{\partial t}+\frac{\partial V_{x} V_{y}}{\partial x}+\frac{\partial V_{y} V_{y}}{\partial y}=-\frac{\partial p}{\partial y}+\frac{\partial 1}{R e}\left(\frac{\partial^{2} V_{y}}{\partial x^{2}}+\frac{\partial^{2} V_{y}}{\partial y^{2}}\right) .
\end{aligned}
$$

Energy equation neglecting viscous dissipation and considering a pure forced convection heat transfer process

$$
\frac{\partial T}{\partial t}+\frac{\partial V_{x} T}{\partial x}+\frac{\partial V_{y} T}{\partial y}=\frac{\partial 1}{R e P r}\left(\frac{\partial^{2} T}{\partial x^{2}}+\frac{\partial^{2} T}{\partial y^{2}}\right) .
$$

Further

$$
R e=\rho U_{\max } d / \mu \text { and } \operatorname{Pr}=c_{p} \mu / k
$$

3.3. Boundary conditions. At inlet: for a parabolic velocity inlet:

$$
\begin{aligned}
& V_{x}=1-(|1-2 \beta y|)^{2} \quad(\text { for } 0 \leq y \leq H / d, \beta=d / H), \\
& v_{x}=0, \quad T=0 .
\end{aligned}
$$

On the surface of the square cylinders, the standard no-slip and constant wall temperature conditions are used:

$$
V_{x}=0, \quad V_{y}=0, \quad T=1 .
$$

On the upper and lower boundaries, the standard no-slip and adiabatic conditions are used:

$$
V_{x}=0, \quad V_{y}=0, \quad \frac{\partial T}{\partial y}=0 .
$$


At the exit boundary

$$
\frac{\partial \phi}{\partial x}=0
$$

where $\phi$ is a dependent variable, $V_{x}, V_{y}$ or $T$.

The output parameters are summarized as follows:

Total drag coefficient, as explained by Patnana et al. [15] for the flow around a circular cylinder, in a horizontal cross flowing domain is as follows:

$$
C_{D}=\frac{F_{D}}{\frac{1}{2} \rho U_{\max }^{2} d}=\int_{S} C_{p} n_{x} \mathrm{~d} S+\frac{2}{R e} \int_{S} \tau n_{x} \mathrm{~d} S
$$

Average Nusselt number:

$$
N u=\frac{h d}{k} .
$$

3.4. Grid generation and solution technique. Figures $2 \mathrm{a}$ and $2 \mathrm{~b}$ display the overall grid structure and close view of the grid structure around the square bluff bodies, respectively. The grid, using quadrilateral cells, generated for this problem is non-staggered in nature. It is generated using the commercial grid generation tool ANSYS Workbench. A finer grid size is maintained near the square cylinders to capture the changes that occur in the flow around the square cylinders. The smallest grid spacing is kept around the square cylinders and confined walls is of $0.008 d$, and the coarsest one is $0.5 d$ which can be seen at the inlet or outlet part of the flowing domain. The number of grid points placed on each surface of the square cylinders are 100, following Durga Prasad and Dhiman [5. The meshing procedure in the zone connecting the square cylinders and the confined walls is done in a manner which can take into account the wall effects in flow process.
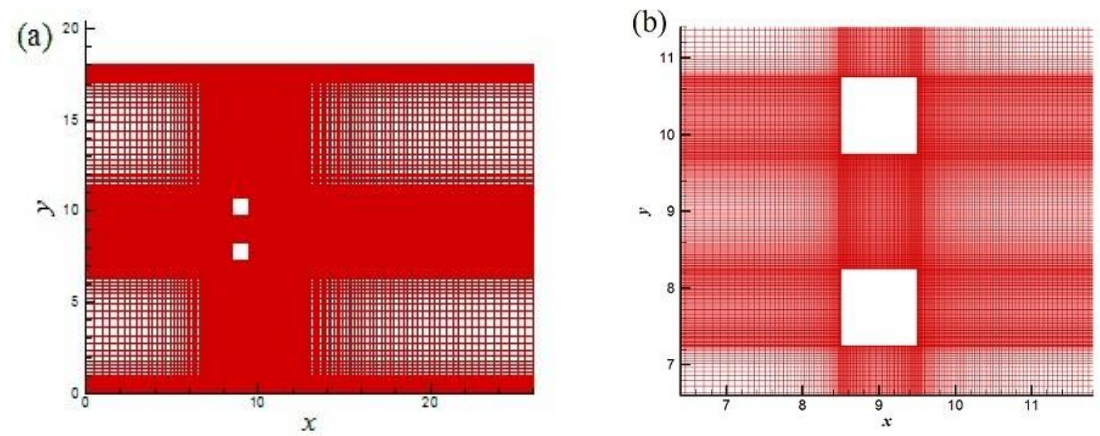

Figure 2. (a) The overall grid structure and (b) close view of the grid structure around the square bluff bodies

Following several books [16, 17, SIMPLE algorithm is used to avoid pressure velocity decoupling and because it offers good convergence for the type of problem under consideration. The absolute convergence criteria is set at $10^{-15}$ for flow parameters and $10^{-20}$ for thermal parameters. Discretization of the convective terms in the momentum and energy equations is done using QUICK, a third-order upwind scheme. 


\section{Results And Discussion}

4.1. Validation. The validation of the results obtained applying the above numerical method was carried out against those of Durga Prasad and Dhiman [5] for the Re ranging from 10 to 40 at $\operatorname{Pr}=0.7$ (resembling air) and 50 (resembling organic polymer liquids) for a constant transverse gap ratio of 1.5 . Tables 1 and 2 show that the values of drag coefficient and Nusselt number stay well within $0.1 \%$ and $3.2 \%$, respectively.

Table 1. Validation of $C_{D}$ with [5] in a steady laminar flow regime

\begin{tabular}{|c|c|c|c|}
\hline $\begin{array}{c}R e \\
(s / d=1.5)\end{array}$ & $\begin{array}{c}C_{D} \text { from } \\
\text { Durga } \\
\text { Prasad and } \\
\text { Dhiman [5] }\end{array}$ & $\begin{array}{c}C_{D} \text { from } \\
\text { current } \\
\text { simulation }\end{array}$ & $\begin{array}{c}\% \\
\text { deviation }\end{array}$ \\
\hline \hline 10 & 3.6034 & 3.6039 & 0.02 \\
\hline 20 & 2.6151 & 2.6153 & 0.01 \\
\hline 30 & 2.2001 & 2.1998 & 0.01 \\
\hline 40 & 1.9646 & 1.9628 & 0.09 \\
\hline
\end{tabular}

Table 2. Validation of Nusselt number with [5] in a steady laminar flow regime

\begin{tabular}{|c|c|c|c|}
\hline $\begin{array}{c}R e \\
(s / d=1.5)\end{array}$ & $\begin{array}{c}N u \text { from } \\
\text { Durga } \\
\text { Prasad and } \\
\text { Dhiman [5] }\end{array}$ & $\begin{array}{c}\text { Nu from } \\
\text { current } \\
\text { simulation }\end{array}$ & $\begin{array}{c}\% \\
\text { deviation }\end{array}$ \\
\hline \hline \multicolumn{4}{|c|}{$\operatorname{Pr}=0.7$} \\
\hline 10 & 1.4856 & 1.4918 & 0.42 \\
\hline 20 & 2.0726 & 2.0913 & 0.90 \\
\hline 30 & 2.4446 & 2.4713 & 1.09 \\
\hline 40 & 2.7402 & 2.7749 & 1.26 \\
\hline \multicolumn{4}{|c|}{$\operatorname{Pr}=50$} \\
\hline 10 & 6.0861 & 6.1320 & 0.75 \\
\hline 20 & 8.4173 & 8.5673 & 1.78 \\
\hline 30 & 10.2586 & 10.4862 & 2.22 \\
\hline 40 & 11.6463 & 12.0215 & 3.20 \\
\hline
\end{tabular}

It was seen that the drag coefficient remained the same for both square cylinders because of the fact that the effect of gravity and the variation of the fluid's density with temperature have been neglected in this problem. The $C_{D}$ values reported above are those of the upper square cylinder in the flow domain. It is also to be noted that the average Nusselt number for both upper and lower square cylinders remains constant owing to similar reasons.

4.2. Fluid flow patterns. Figure 3 shows the streamlines at $s / d=1.5$ for $R e=20$ and 40. The flow is found to be steady in this flow range and at the same time an anti-phase pattern (wake structures generated from both square cylinders are equal 

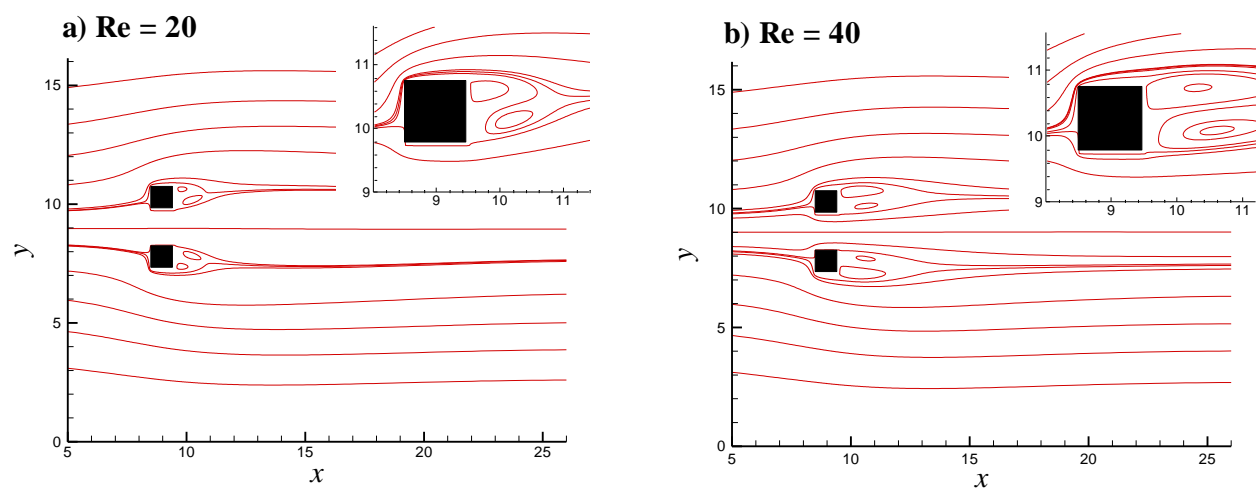

Figure 3. (a-b) Streamlines along with the magnified views of upper square cylinder for a transverse gap ratio of 1.5

and oppositely directed in a given plane) is seen. This pattern gradually glorifies as $R e$ is increased from 20 to 40, which clearly depicts the approaching unsteadiness in the downstream.

A close look at the magnified image of the streamline contours reveals the formation of wakes at the rear part of the square bluff bodies, which widen with increasing $R e$. Further, it is to be noted that there is no possibility of reverse flow in the domain. These streamlines also show a marginal interference of stream functions due to the presence of two square bluff bodies. The transverse gap ratio falls under that needed to produce a quasi-periodic flow regime in accordance with results from previous studies [1].

4.3. Thermal patterns. Figure 4 shows the isotherm contours of the fluid flowing past the pair of side-by-side square bluff bodies at $R e=20$ and 40 for the $\operatorname{Pr}=0.7$ and 50. Following Merkin 18, who stated that during the flow process, cooling a cylinder brings about separation near the stagnation point, this is also evident in this case by the clusters of isotherms accumulating in front of the frontal surface of the square bluff bodies. Despite the fact that the domain and flow structures are different from Merkin [18, the concept of high heat transfer in the front part of the obstacle stays intact irrespective of domain. In terms of magnitude factor, the results will always vary with configurations. The observation of the above pattern remains the same in all the cases in the direction of flow. This eventually leads to an increased Nusselt number (heat transfer rate) at the front surfaces compared to that of other surfaces. In fact one can also conclude that the heat transfer is maximum in the front surface followed by an intermediate degree on surfaces parallel to the flow and the rear face has the lowest heat transfer rate. The isotherms also seem to be steady and symmetric along the centerline with almost no interaction at $\operatorname{Pr}=50$, but the interaction prevails at $\operatorname{Pr}=0.7$. This factor can be explained from the concept of boundary layer 

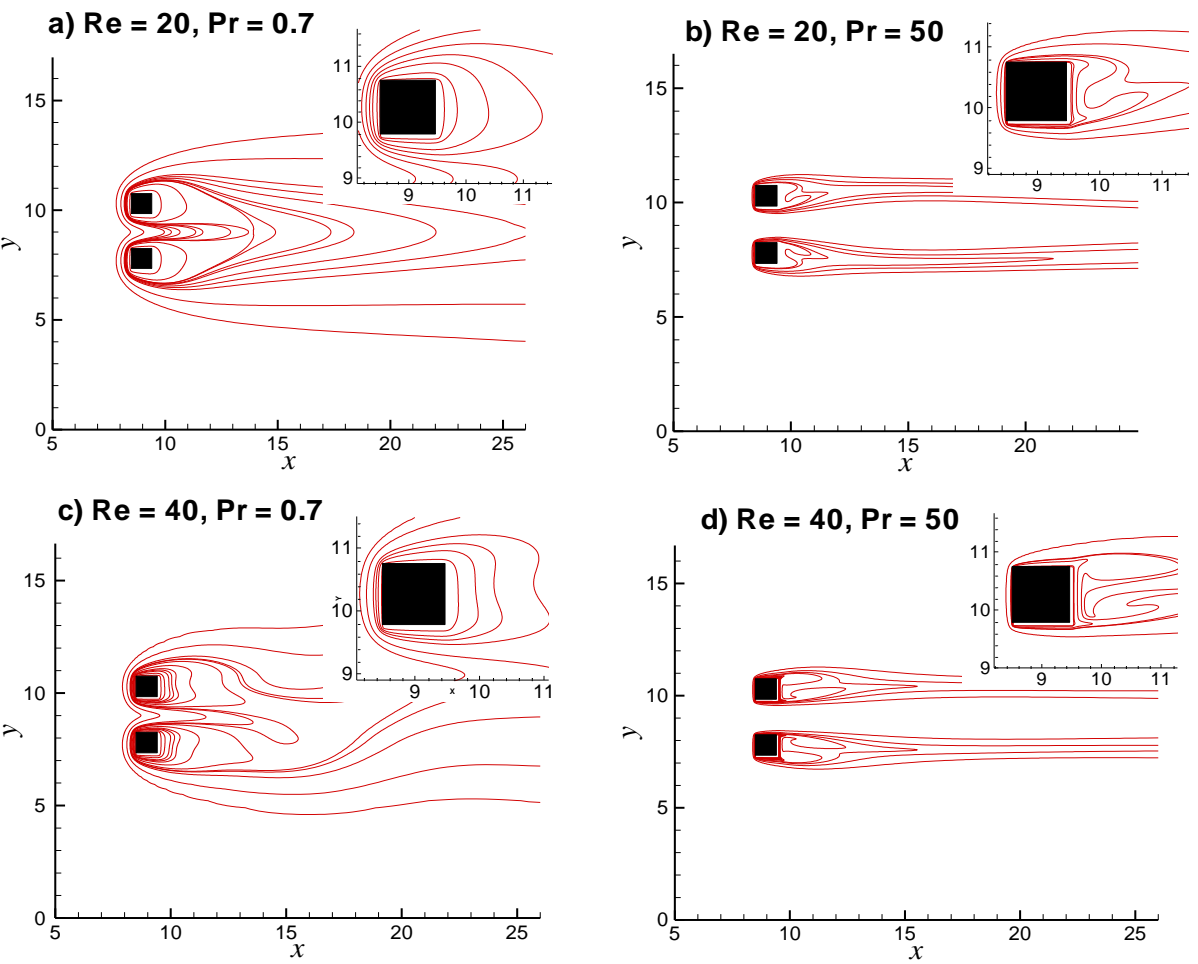

Figure 4. Isotherms along with the magnified views of upper square cylinder for a transverse gap ratio of 1.5

theory, where $\operatorname{Pr}=0.7$ indicates that the hydrodynamic boundary layer is smaller than the thermal boundary layer, which means that the layers tend to move outward which in turn leads to interaction of isotherms due to the presence of the two square cylinders. But at $\operatorname{Pr}=50$, the thermal boundary layer is smaller than that of its hydrodynamic counterpart, as a result, the isotherms tend to die down at a close distance in the downstream from the rear surface of the square cylinders.

The magnified views of the isothermal contours also demonstrates that the wakes formed at $R e=20$ gradually increase in size at $R e=40$. These figures also explain clustering of isotherms near the rear surfaces of the square cylinders, which increases with increasing Reynolds and Prandtl numbers.

4.4. Recirculation length. This is the distance from the rear surface of the obstacle to the point of attachment for the near closed streamline on the axis of symmetry. Figure 5 shows that the recirculation length varies linearly with increasing $R e$ (Table 3). It increases with $R e$ and the results fit linearly with a mere $0.001 \%$ deviation. 
Table 3. Variation of recirculation length with Reynolds number

\begin{tabular}{|c|c|}
\hline$R e$ & Lr \\
\hline \hline 10 & 0.4276 \\
\hline 20 & 1.0039 \\
\hline 30 & 1.6146 \\
\hline 40 & 2.2406 \\
\hline
\end{tabular}

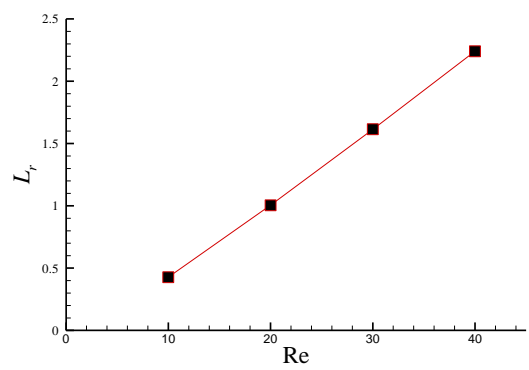

Figure 5. Variation of recirculation length with Reynolds number

The following simple correlation is established for the calculation of wake length $\left(L_{r}\right)$, for the intermediate values of physical parameters in the steady confined regime:

$$
L_{r}=0.06 R e-0.19 \quad \text { for } 10 \leq R e \leq 40
$$

This linearity in recirculation length versus $R e$ plot has also been observed by Sharma et al. [19] for a single square cylinder.

Further, the variation in recirculation length with the change in transverse gap ratio was studied with $s / d$ varying from 0.7 to 10 for $R e=40$. The recirculation length is expected to be quite pronounced as compared to the lesser $R e$ values, and at the same time to maintain the steadiness in the flow. The trends observed from Table 4 show that the recirculation length increases initially with increase in $s / d$ to a maximum value beyond which the value decreases with the increase in $s / d$. Small gap ratio involves chaotic interactions of wake generated from both square cylinders, thereby reducing the wake length but as the transverse gap ratio increases the wake formations are subjected to minimum hindrance from the other body. But with further increase in $s / d$, a decrease in wake length is observed, which accounts for a hindrance evolving out of the confined walls.

Table 4. Variation of recirculation length with transverse gap ratio $(s / d)$ at $R e=40$

\begin{tabular}{|c|c|}
\hline$s / d$ & $L_{r}($ at $R e=40)$ \\
\hline \hline 0.7 & 1.3083 \\
\hline 1.5 & 2.2406 \\
\hline 2.5 & 2.2035 \\
\hline 5.0 & 2.1983 \\
\hline 10.0 & 1.4395 \\
\hline
\end{tabular}



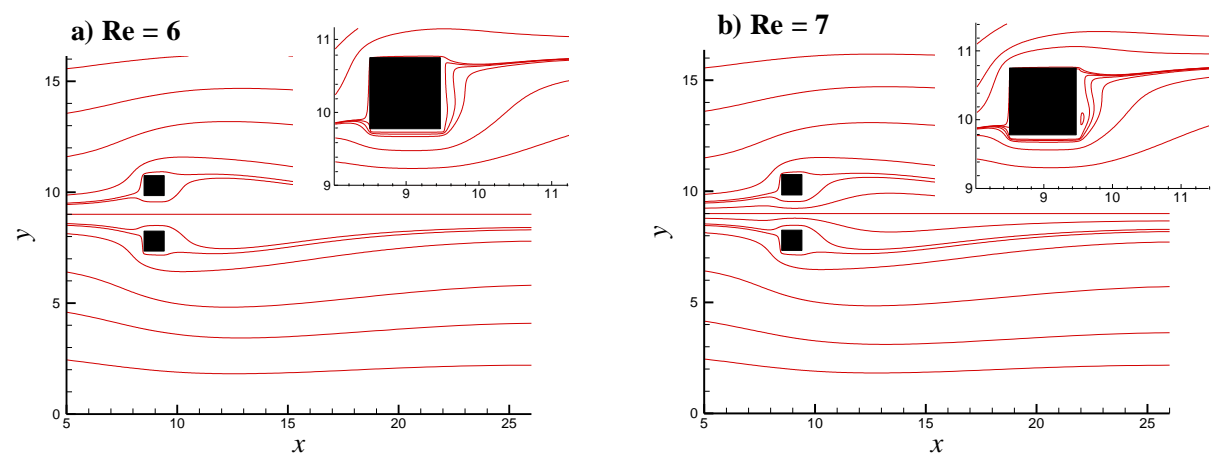

Figure 6. Streamlines along with magnified view of upper square cylinder showing absence of any wake at $R e=6$ and the onset of wake formation at $R e=7$

Furthermore, the onset of the flow separation is determined. It has been observed that there is no presence of recirculation wake at $\operatorname{Re}=6$, whereas recirculation commences at $\operatorname{Re}=7$ (as shown in Figures 6a and 6b, respectively). Hence, one can infer that a higher the magnitude of viscous dominance in the flow field, the lower or minimal is the recirculation or wake length formed at the rear side of the square cylinders.

\section{Conclusions}

Summarizing the present study, one can make the observation that the drag coefficient increases with the increase in $R e$, whereas $N u$ increases with the increase in both $R e$ and $\mathrm{Pr}$ as is evident from the stream function and isothermal contours. It has also been seen that the recirculation length increases linearly with the increase in $R e$ in steady laminar flow regime $(R e=10$ to 40$)$. This paves a way for further determination of recirculation length for various flow regimes for the flow over a pair of side-by-side square cylinders. One can also correlate the results with that their cylindrical counterpart thereby leading to the appropriate justification of choice of obstacle shape during various industrial operations. The effect of $s / d$ on $L_{r}$ is determined and the $L_{r}$ correlation with respect to varying $R e$ has been identified. Finally, the onset of flow separation is determined for the current framework and it occurs at $R e=7$.

Acknowledgement. We thank editors and reviewers for their valuable comments that greatly improved the manuscript.

\section{REFERENCES}

1. Valencia, A. and Paredes, R.: Laminar flow and heat transfer in confined channel flow past square bars arranged side-by-side. Heat and Mass Transfer, 39, (2003), 721-728. 
2. Peng, Y. F.: On the bi-stabilities of vortex shedding flows behind a pair of square solids. Journal of Chinese Institute Engineers, 27, (2004), 385-393.

3. Agrawal, A. Djenidi, L. and Antonia, R. A.: Investigation of flow around a pair of side-by-side square cylinders using the lattice Boltzmann method. Computers and Fluids, 35, (2006), 1093-1107.

4. RAO, Y. Ni, Y. and LiU, C.: Flow effect around two square cylinders arranged side-byside using lattice Boltzmann method. International Journal of Modern Physics $C, \mathbf{1 9}$, (2008), 1683-1694.

5. Durga Prasad, A. V. V. S. and Dhiman, A. K.: CFD analysis of momentum and heat transfer around a pair of square cylinders in side-by-side arrangement. Heat Transfer Engineering, 35, (2014), 398-411.

6. Wong, P. T. Y., Ko, N. W. M. and ChiU, A. Y. W.: Flow characteristics around two parallel adjacent square cylinders of different sizes. Journal of Wind Engineering and Industrial Aerodynamics, 54/55, (1995), 263-275.

7. Kolar, V., Lyn, D. A. and Rodi, W.: Ensemble-averaged measurements in the turbulent near wake of two side-by-side square cylinders. Journal of Fluid Mechanics, 346, (1997), 201-237.

8. Alam, M. M., Zhou, Y. and Wang, X. W.: The wake of two side-by-side square cylinders. Journal of Fluid Mechanics, 669, (2011), 432-471.

9. Harichandan, A. B. and Roy, A.: 2012, Numerical investigation of flow past single and tandem cylindrical bodies in the vicinity of a plane wall. Journal of Fluids and Structures, 33, (2012), 19-43.

10. Mizushima, J. and Akinaga, T.: Vortex shedding from a row of square bars. Fluid Dynamics Research, 32, (2003), 179-191.

11. Kumar, S. R., Sharma, A. and Agrawal, A.: Simulation of flow around a row of square cylinders. Journal of Fluid Mechanics, 606, (2008), 369-397.

12. Sewatkar, C. M., Sharma, A. and Agrawal, A.: On the effect of Reynolds number for flow around a row of square cylinders. Physics of Fluids, 21, (2009), 083602108360213.

13. Chatterjee, D., Biswas, G. and Amiroudine, S.: Numerical simulation of flow past row of square cylinders for various separation ratios. Computers and Fluids, 39, (2010), 49-59.

14. Chhabra, R. P. and Richardson, J. F.: Co-current horizontal and vertical upwards flow of gas and non-Newtonian liquid, Encyclopedia of Fluid Mechanics, Vol. 3, Edited by N. P. Cheremisinoff, Gulf, Houston (USA), (1986), 563-609.

15. Patnana, V. K., Bharti, R. P. and Chhabra, R. P.: Two-dimensional unsteady flow of power-law fluids over a cylinder. Chemical Engineering Science, 64, (2009), 2978-2999.

16. Muralidharan, K. and Sundararajan, T.: Computational Fluid Flow and Heat Transfer. 2nd edition, Narosa publications, 2000.

17. Anderson, J. D. Jr.: Computational Fluid Dynamics - the Basics with Applications. 2nd edition, McGraw Hill publication, 2000.

18. Merkin, J. H.: Mixed convection from a horizontal circular cylinder. International Journal of Heat and Mass Transfer, 20, (1977), 73-77. 
19. Sharma, N. Dhiman, A. K. and Kumar, S.: Mixed Convection flow and heat transfer across a square cylinder under the influence of aiding buoyancy at low Reynolds numbers. International Journal of Heat and Mass Transfer, 55, (2012), 2601-2614. 